\title{
Oncology Nurses Knowledge and Attitudes Regarding Cancer Pain Management
}

\author{
Shahdad Shahriary ${ }^{1}$, Seyed Mostafa Shiryazdi*, Seyed Ali Shiryazdi², Amir \\ Arjomandi' $^{2}$, Fatemeh Haghighi ${ }^{3}$, Fariba Mir Vakili ${ }^{3}$, Naiemeh Mostafaie ${ }^{3}$
}

\begin{abstract}
Background: Oncology nurses play a crucial role in cancer pain management and must be highly informed to ensure their effective practice in the cancer setting. The aim of this study was to determine the baseline level of knowledge and attitudes of oncology nurses regarding cancer pain management. Materials and Methods: A cross-sectional survey research design was employed. The sample comprised 58 cancer nurses working in Shahid Sadoughi hospital, Yazd, Iran. The "'Nurses Knowledge and Attitudes Survey Regarding Pain” (NKAS) tool and a demographic form were utilized to ascertain the knowledge and attitudes of oncology nurses working in oncology settings. Results: The average correct response rate for oncology nurses was $66.6 \%$, ranging from $12.1 \%$ to $94.8 \%$. The nurses mean score on the knowledge and attitudes survey regarding pain management was $28.5 \%$. Results revealed that the mean percentage score overall was $65.7 \%$. Only $8.6 \%$ of nurse participants obtained a passing score of $\mathbf{7 5 \%}$ or greater. Widespread knowledge deficits and poor attitudes were noted in this study, particularly regard pharmacological management of pain. Conclusions: The present study provides important information about knowledge deficits in pain management among oncology nurses and limited training regarding pain management. Our results support the universal concern of inadequate knowledge and attitudes of nurses regarding cancer pain. It is suggested educational and quality improvement initiatives in pain management could enhance nurses knowledge in the area of pain and possibly improve practice.
\end{abstract}

Keywords: Pain management - oncology nurses - cancer units - knowledge - attitudes

Asian Pac J Cancer Prev, 16 (17), 7501-7506

\section{Introduction}

Cancer pain can have various causes ranging from direct tumor invasion, metastasis, and other disease processes to pain that is not related to the cancer diagnosis (Eftekhar et al., 2007; Yaakup et al., 2014). Direct tumor involvement is the most common cause of cancer pain, representing about two-thirds of cancer pain (Cleary et al., 2000). Pain is a prevalent problem for the majority of those hospitalized for cancer and patients. The survey data on the incidence and prevalence and of cancer and cancer-related pain indicate that a majority of patients experience pain at one time or another during the course of treatment and that cancer pain impairs quality of life and functionality (Agency for Healthcare Research and Quality, 2002). Pain is experienced by $30 \%$ to $50 \%$ of cancer patients receiving treatment and by $70 \%$ to $90 \%$ of patients with metastatic or advanced disease. Estimates of the incidence of pain in hospitalized cancer patients have been reported as high as $90 \%$ (Nersesyan et al., 2007).

The main goal of nursing care is the promotion of optimal wellness of the individual through maintenance, or attainment, of system stability by strengthening the lines of resistance. One barrier in the management of cancer pain is the lack of knowledge on the part of healthcare providers, including staff nurses (Arslan et al., 2014; Lee et al., 2014). The Oncology Nursing Society's (ONS) position on cancer pain makes it clear that all people have the right to optimal pain relief, which includes culturally relevant and sensitive pain education, assessment, and management (American Pain Society, 2005; Daher et al., 2010). In addition, The Joint Commission on Accreditation of Healthcare Organizations (JCAHO, 2000) standard asserts that patients have the right to appropriate assessment and management of pain, and that it is the staff's responsibility to respect and support each patient's right to pain management. According to the JCAHO, 2000, the majority of nurses are not meeting JCAHO standards of care in the management and documentation of cancerrelated pain which can interfere with care and have legal implications (JCAHO, 2000).

The management of pain by nurses was under scrutiny because of reports of inadequate pain relief documented in the literature. According to the previous reports, patients' pain management is being inadequately managed and documented by nurses across. Inadequate

${ }^{1}$ Baghaie-pour Clinic, ${ }^{2}$ Department of General Surgery, ${ }^{3}$ Department of Gynecology, Shahid Sadoughi University of Medical Sciences and Health Services, Yazd, Iran *For correspondence: seyedmostafashiryazdi@gmail.com, seyedmostafashiryazdi@gmail.com 
pain management has serious consequences for the patient, physician, nurse, and the health care system. Therefore, in this study we have tried to survey oncology nurses knowledge and attitude regarding cancer pain management.

\section{Materials and Methods}

This cross-sectional study invited all registered nurses and licensed practical nurses working on oncology units in a teaching hospital to complete a questionnaire on their knowledge and attitudes regarding cancer pain. Shahid Sadoughi Hospital is one of the largest teaching hospitals in south west of Iran. We invited oncology nurses to participate in the study because they are very often the first individuals to assess a pain complaint. If these staff did not respond appropriately, effective pain management could be delayed or omitted. Eligibility criteria included all registered and enrolled nurses working part-time and full-time in the cancer units and exclusion criteria included the registered and enrolled nurses who was/were on annual leave more than two weeks and only on night shift during the data collection period.

A letter explaining the purpose of the study, as well as the inclusion criteria for participation, was given to each participant. The letter also stated that filling out the questionnaires implied consent, and that participation was confidential and voluntary. The researcher ensured that the confidentiality of each individual respondent was maintained throughout the course of the research study. No form of personal identification was incorporated onto the survey, therefore, confidentiality and anonymity of the respondents was guaranteed. The researcher had no mechanism to identify individual respondents.

\section{Instrument}

A self-administered questionnaire including demographics and Nurses' Knowledge and Attitude Survey Regarding Pain (KAS) was distributed to nurses working on the oncology units. Nurses' Knowledge and Attitude Survey Regarding Pain (KAS) was developed and validated by Ferrell McCaffery in 1987 (Ferrell and McCaffery, 1995). The KAS was a 39-item instrument included aspects of pain assessment, pharmacologic and non-pharmacologic interventions, and attitude about pain management. The content of the KAS was derived from pain management guidelines and standards including those of American Pain Society, The World Health Organization (WHO) and the U.S. Agency for Health Care Policy and Research (AHCPR).

The scoring system of KAS is by analyzing the data in terms of the overall percentage of correct scores as well as in analyzing individual items. All items are equally weighted with the maximum possible score being 39 . Correct responses are given a value of one and an incorrect or blank response was given a value of zero. Therefore, a higher score indicates higher number of correct responses on the survey.

The first 22 questions on the Nurses' Knowledge and Attitudes Survey Regarding Pain are 'True" or 'False" and respondents are asked to circle the best answer.
This is followed by 13 multiple- choice questions. The responses were measured on a Likert-type (summated rating) scale with each item ranging from strongly disagree (SD), disagree (D), agree (A), to strongly agree (SA). The possible range for scores is 25 to 100 , with higher scores reflecting more positive attitudes. In the last part of the survey, two case studies are presented, with each case study followed by two multiple choice questions. According to the authors of this survey, they found that it is most helpful to avoid distinguishing items as measuring either knowledge or attitudes, since many items really measure both. Therefore, data are analyzed in terms of the percentage of complete scores as well as in analyzing individual items.

\section{Statistical analysis}

Data Analysis Demographic data was analyzed using descriptive statistics, such as frequencies, percentages, means and standard deviations. Microsoft Excel, as well as, the Statistical Package for Social Sciences (SPSS) was utilized to analyze the level of knowledge nurses and their attitudes towards pain management.

\section{Results}

Table 1 shows the demographic characteristics of oncology nurses. The sample for this study consisted of 62 oncology nurses which all of the nurses were female. Participants in this descriptive, exploratory study were 53 nurses from three oncology units. The mean age of nurses was 33.5 years with a range from 25 to 48 years. The majority of participants stated that they were full time nursing with no jobs $(82.7 \%, \mathrm{n}=48)$, while $17.24 \%$ $(n=10)$ were working as certified nursing assistants and nurse technicians. In terms of the education level, most of the respondents had a Baccalaureate $(94.8 \%)$. Although, in the area of pain management, $13.8 \%(n=8)$ of nurses had pain management training, while $80 \%(n=34)$ had no training at all other than that give in their nursing program.

Table 2 shows the number, percentage and rank of correct answer for each item of the KAS. A total of 62 Pain questionnaire packages were delivered across the 3 medical units, and 58 questionnaires were returned, a response rate of $95.1 \%$. Of the returned questionnaires,

Table 1. Demographic Characteristics of Oncology Nurses

\begin{tabular}{lcc}
\hline Variables & Frequency $(\mathrm{N})$ & Percentage (\%) \\
\hline Age & 46 & 79.3 \\
$25-40$ & 12 & 20.7 \\
$41-50$ & & \\
Level of Education & & \\
Baccalaureate & 55 & 94.8 \\
Masters or higher & 3 & 5.2 \\
Experience & 14 & 24.2 \\
$\quad<1$ year & 38 & 65.5 \\
1-5 years & 6 & 10.2 \\
5-10 years & & \\
Previous training & 8 & 13.8 \\
Yes & 50 & 86.2 \\
No &
\end{tabular}




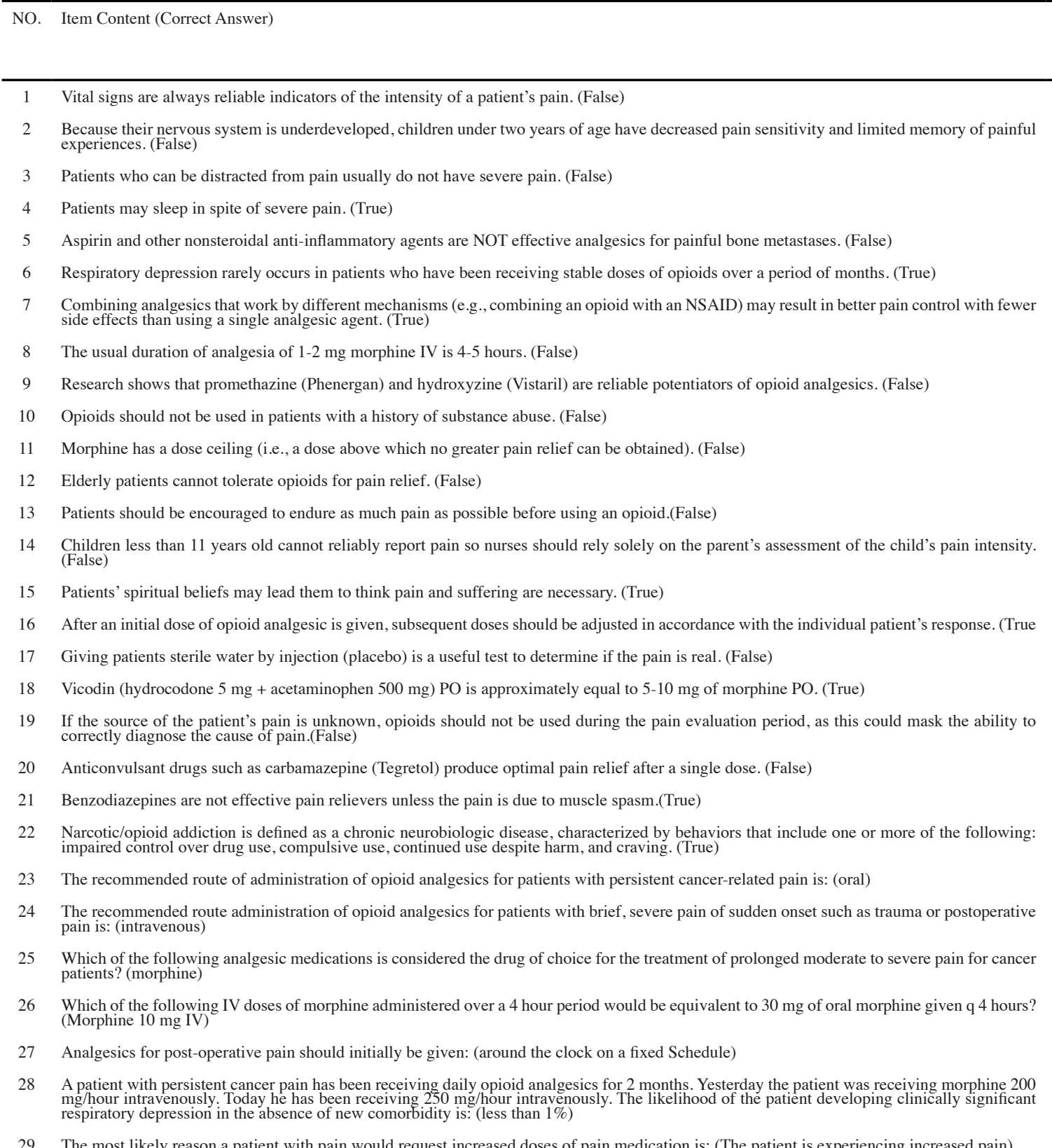

2 Because their nervous system is underdeveloped, children under two years of age have decreased pain sensitivity and limited memory of painful

Patients who can be distracted from pain usually do not have severe pain. (False) 30 (51.8)

29 The most likely reason a patient with pain would request increased doses of pain medication is: (The patient is experiencing increased pain)

30 Which of the following is useful for treatment of cancer pain? (All of the above)

31 The most accurate judge of the intensity of the patient's pain is: (the patient)

32 Which of the following describes the best approach for cultural considerations in caring for patients in pain: (Patients should be individually assessed to determine cultural influences)

33 How likely it is those patients who develop pain already have an alcohol and drug abuse problem? (5-15\%)

34 The time to peak effect for morphine given IV is: (15 min.)

35 The time to peak effect for morphine given orally is: (1.5 - 2 hours).

36 Following abrupt discontinuation of an opioid, physical dependence is manifested by the following: (sweating, yawning, nausea and vomiting when the opioid is abruptly discontinued)

37a Patient A: Andrew is 25 years old and this is his first day following abdominal surgery. As you enter his room, he smiles at you and continues $=$ no pain/discomfort, $10=$ worst pain/discomfort) he rates his pain as $8 . \mathrm{A}$
Circle the number that represents your assessment of Andrew's pain. (8)

37b Your assessment, above, is made two hours after he received morphine $2 \mathrm{mg}$ IV. Half hourly pain ratings following the injection ranged from 6 to 8 and he had no clinically significant respiratory depression, sedation, or other untoward side effects. He has identified $2 / 10$ as an acceptable level of pain relief. His physician's order for analgesia is "morphine IV 1-3 mg q1h PRN pain relief." Check the action you will take at this time. (Administer morphine $3 \mathrm{mg}$ IV now)

38a Patient B: Robert is 25 years old and this is his first day following abdominal surgery. As you enter his room, he is lying quietly in bed and grimaces as he turns in bed. Your assessment reveals the following information: $\mathrm{BP}=120 / 80 ; \mathrm{HR}=80 ; \mathrm{R}=18$; on a scale of 0 to $10(0=$ no pain $/$ discomfort, $10=$ worst pain/discomfort) he rates his pain as 8 . A. On the patient's record you must mark his pain on the scale below. Circle the number that represents your assessment of Robert's pain: (8)

38b Your assessment, above, is made two hours after he received morphine $2 \mathrm{mg}$ IV. Half hourly pain ratings following the injection ranged from 6 to 8 and he had no clinically significant respiratory depression, sedation, or other untoward side effects. He has identified $2 / 10$ as an acceptable level of pain relief. His physician's order for analgesia is "morphine IV 1-3 mg q1h PRN pain relief." Check the action you will take at this time: (Administer morphine $3 \mathrm{mg}$ IV now) 
only 1 participant failed to complete the questionnaire or had left pages unanswered and therefore were withdrawn from the study, resulting in 58 participants, a final response rate of $93.5 \%$.

The average correct response rate for oncology nurses was $66.6 \%$, ranging from $12.1 \%$ to $94.8 \%$. The mean total correct score of the KAS was 28.5 (range=21-32; S.D=3.41). 53 out of the KAS items were answered incorrectly by at least half of the sample.

The highest percentages of correct answers were for items 37b, 38b (94.8\%), and 36 (89.6\%). Among the 40 items surveyed, 20 items had a correct answer rate of higher than $70 \%(1-4,8,14,15,22-24,27,29,31$, $33,35,36,37 \mathrm{a}, 37 \mathrm{~b}, 38 \mathrm{a}$, and 38b). 9 items received a correct answer rate of less than 50\% (5, 9-11, 16, 18, 19, 25, and 34).

The five items that the respondents most frequently answered correctly were in relation to the pain tolerance of different age groups and monitor patients' response of pain management.

\section{Discussion}

Pain is a prevalent problem for the majority of those hospitalized for cancer and patients' satisfaction with their pain management can improve when patients feel that their pain needs are being addressed (Sternman et al., 2003). Despite policy advances in cancer pain management and improvements in radiological and surgical treatments, and the efficacy of current pharmacology, research suggests that many patients with cancer continue to experience high levels of pain (Kassa et al., 2014). It is reported that $44 \%$ of cancer patients were experiencing moderate to greater than moderate pain, but only $41 \%$ of those patients experiencing pain were unsatisfied with their pain management. Findings have prompted recent researchers to look more roughly at organizational and behavioral factors that may be contributing to the cancer pain problem. There is a growing body of evidence which noted that unrelieved cancer pain may be more the result of patients' and health care providers' beliefs and attitudes, rather than the lack of effective pain treatment (Clarke et al., 1996).

Our study provides important information about the level of oncology nurses knowledge and attitude towards cancer pain in our hospital. The study results showed that nurses' knowledge about pain management was far from optimal. The majority of the nurses in this study answered less than $80 \%$ of the questions correctly indicating that nurses continued to lack sufficient knowledge regarding pain management. These results are comparable with studies in Italy, Hong Kong and Saudi Arabia oncology nurses (Bernardi et al., 2007; Lui et al., 2008; Yildirim et al., 2008; Tufekci et al., 2013; Alqahtani et al., 2015). These results show that Iranian nurses have answered similarly to other studies among special groups of nurses (Clarke et al., 1996; McCaffery and Ferrell, 1997; Visentin et al., 2001; McCaffery et al., 2002; Tafas et al., 2002; Lai et al., 2003). Bernardi et al., 2007 have showed that more than 50\% of oncology nurses underestimated the pain of patients and they did not treat it in a correct way and they had an incorrect self-evaluation about their pain management knowledge. However, Tufekci et al., 2013 in turkey shown that nurses' pain knowledge was significantly different according to the clinics in which they worked $(\mathrm{p}<0.001)$. However, Rushton et al., 2003 studied knowledge and attitudes about cancer pain management among oncology and non-oncology nurses. Their findings suggested that the oncology nurses knowledge correlated more closely with the recommended principles of pain management in the cancer patient than did those of the non-oncology nurses (Rushton et al., 2003). Wilson et al., 2007 have suggested that the hospice/oncology nurses' had a more comprehensive knowledge of pharmacology, theories of pain, and management. Interestingly, the knowledge of those nurses did not appear to be related to their years of experience (Wilson et al., 2007).

The correct answer rate for the entire scale on the NKAS survey in this present study was $65.7 \%$ with a range of between $41.0 \%-84.6 \%$. Previous studies have reported means of between $35 \%$ and $79 \%$ on the NKAS survey. The mean score obtained in this present study compares well with other similar studies (Bernardi et al., 2007; Lui et al., 2008; Yildirim et al., 2008; Tufekci et al., 2013; Alqahtani et al., 2015). There are concern regarding the safe administration of opioid medication to elderly patients, it can be tolerated in lower doses by geriatric patients (Smeltzer et al., 2008:268). However, in this study According to $32.2 \%$ respondents believed that elderly patients cannot tolerate opioids for pain relief.

Children's pain also is compounded by the following factors: constant development, relatively limited cognitive ability, limited verbal skills, and limited behavioral competencies. Only $63.7 \%$ of oncology nurses in this study stated that they believed children who reported pain. This response was similar other studies, which ranged from $60 \%$ to $75 \%$ (Bernardi et al., 2007; Tufekci et al., 2013). Nurses do not believe pain reported by children because they might think that children's understanding of pain is limited.

In this study, only $18.9 \%$ of nurses achieved a score of $80 \%$ or greater on the NKAS survey which indicates the magnitude of knowledge deficits and poor attitudes. However, Lewthwaite et al. 2011 reported that in their study nearly half of the nurses achieved a score of $80 \%$ or greater (Lewthwaite et al., 2011).

It is reported that nurses remained less likely to relieve the pain of a smiling patient than that of a grimacing patient, regardless of the patient's own report of pain. This showed the need for the improved and consistent nursing education regarding proper pain management techniques in the hospital setting (McCaffery et al., 1999). Although nurses are not responsible for the prescription of pain medicine, the decision to administer or to withhold pain medications is well within the nurses practices (Bernhofer et al., 2011). It seems that nurse's deficient beliefs, attitudes or knowledge about pain management can affect the level of pain experienced by a patient while in the medical settings (McCafferyet al., 1997).

It is reported that the unwarranted fear of contributing to the patient's addiction as integral segment of the nurses' knowledge deficit (Aziato et al., 2014). Studies have 
shown that the main deficiencies for nurses in different specials relate to the correct use of analgesic drugs, pharmacological properties of opioids and addiction. The majority of nurses were unable to identify equianalgesic doses for some common analgesics. In our study, the highest percentages of incorrect answers for all of nurses were noted in relation to the pain medication items including: reliability of Phenergan and Vistaril, 18.9\%; Morphine's dose ceiling, 20.7\%; equality between the dose of Vicodin PO and morphine PO, 12.1\%; situation where opioids should not be used, $13.8 \%$; and Opioids using, 27.5\%).

Previous studies identified some barriers to lack of nurses' knowledge about pain management included lack of time, workload, reluctance of clinicians to prescribe analgesia, and the lack of nursing knowledge regarding opioid administration (Pretorius et al., 2014). When major medical and nursing textbooks published worldwide are examined, it is seen that pain makes up a small part of the content (Ferrell et al., 2000) and it has been reported that baccalaureate nursing programs allocate little time to pain management (O'Brien et al., 1996). Similarly, It seems inadequate pain knowledge of the nurses may be the lack of emphasis on pain management in formal nursing education in Iran. There are no standards for the duration and content of pain management courses in Iran nursing courses. It seems that oncology nurses have not the same education in pain management across Iran due different courses that they passed in the schools. It is necessary that a more extensive information program for nurses should be carried out in university and continuing education course. Education Meanwhile, it is reported that nurses' knowledge and attitudes in cancer pain management initially improved after educational intervention, but then declined over time (Goldberg et al., 2007).

In conclusion, This study addressed the inadequacy of knowledge and incorrect attitudes existed in pain management of oncology nurses. As with the previous studies, the findings suggest oncology nurses have a lack of knowledge in regard to the pharmacology of medications used in managing cancer pain. Therefore, the findings suggested the need for improvements for effective pain management, recommendation provided concerns about the educational need and together with the interventions of the health care systems for optimal relief of pain. It is proposed that the nursing curriculum should include compulsory pain courses and national course programs about effective pain management.

\section{References}

Alqahtani M, Jones LK (2015). Quantitative study of oncology nurses' knowledge and attitudes towards pain management in Saudi Arabian hospitals. Eur J Oncol Nurs, 19, 44-9.

American Nurses Society (2005). ANCC announces new certification for pain management nurses. Retrieved January 15, 2010 from: http://www.nursingworld. org/FunctionalMenuCategories/MediaResources/ PressReleases/2005/pr09208536.aspx.

American Pain Society. (2005). Guideline for the management of cancer pain in adults and children. Glenview, IL.

Arslan D, Koca T, Akar E, et al (2014). Cancer pain prevalence and its management. Asian Pac J Cancer Prev, 15, 8557-62.

Aziato L, Adejumo O (2014). Determinants of nurses' knowledge gap on pain management in Ghana. Nurse Educ Pract, 14, 195-9.

Bernardi M, Catania G, Tridello G (2007). Knowledge and attitudes about cancer pain management: a national survey of Italian hospice nurses. Cancer Nurs, 30, 20-6.

Bernhofer E (2011). Ethics: ethics and pain management in hospitalized patients. Online J Issues Nurs, 17, 11 .

Clarke EB, French B, Bilodeau ML, et al (1996). Pain management knowledge, attitudes and clinical practice: the impact of nurses' characteristics and education. J Pain Symptom Manage, 11, 18-31.

Daher M (2011).. Pain relief is a human right. Asian Pac $J$ Cancer Prev, 11, 97-101.

Eftekhar Z, Mohaghegh MA, Yarandi F, et al (2014). Knowledge and attitudes of physicians in Iran with regard to chronic cancer pain. Asian Pac J Cancer Prev, 8, 383-6.

Ferrell BR, Grant M, Ritchey KJ, et al (1993). The pain resource nurse training program: a unique approach to pain management, J Pain Symptom Manage, 8, 549-56.

Fife BL (1993), A comparative study of the attitudes of physicians and nurses toward the management of cancer pain. J Pain Symptom Manage, 8, 132-9.

Goldberg GR, Morrison RS (2007). Pain management in hospitalized cancer patients: a systematic review. J Clin Oncol, 25, 1792-801.

Joint Commission on Accreditation of Healthcare Organizations (2000). Comprehensive Accreditation Manual for Hospitals: The Official Handbook. Oakbrook Terrace, IL: Joint Commission Resources.

Kassa H, Murugan R, Zewdu F, et al (2014). Assessment of knowledge, attitude and practice and associated factors towards palliative care among nursesworking in selected hospitals, Addis Ababa, Ethiopia. BMC Palliat Care, 13, 6.

Lee YJ, Hyun MK, Jung YJ, et al (2014). Effectiveness of education interventions for the management of cancer pain: a systematic review. Asian Pac J Cancer Prev, 15, 4787-93.

Lewthwaite BJ, Jabusch KM, Wheeler BJ, et al (2011). Nurses' knowledge and attitudes regarding pain management in hospitalized adults. J Contin Educ Nurs, 42, 251-7.

Lui LY, So WK, Fong DY (2008). Knowledge and attitudes regarding pain management among nurses in Hong Kong medical units. J Clin Nurs, 17, 2014-21.

Management of Cancer Symptoms: Pain, Depression, and Fatigue. Summary, Evidence Report/Technology Assessment: Number 61. AHRQ Publication No. 02-E031, July 2002. Agency for Healthcare Research and Quality, Rockville, MD.

McCaffery M, Ferrell BR (1995). Nurses' knowledge about cancer pain: A survey of five countries. J Pain Symptom Manage, 10, 356-69.

McCaffery M, Ferrell BR (1997). Influence of professional vs. personal role on pain assessment and use of opioids. J Contin Educ Nurs, 28, 69-77.

McMillan SC, Tittle M, Hagan S, et al (2000). Knowledge and attitudes of nurses in veterans hospitals about pain management in patients with cancer. Oncol Nurs Forum, 27, 1415-23.

Nersesyan H, Slavin KV (2007). Current approach to cancer pain management: Availability and implications of different treatment options. Ther Clin Risk Manag, 3, 381-400.

O'Brien S, Dalton JA, Konsler G, et al (1996). The knowledge and attitudes of experienced oncology nurses regarding the management of cancer-related pain. Oncol Nurs Forum, 23, 515-21.

Pretorius A, Searle J, Marshall B (2014). Barriers and enablers to emergency department nurses management of patient's 
Shahdad Shahriary et al

pain. Pain Manag Nurs, S1524-9042, 146-5.

Rushton P, Eggett D, Sutherland CW (2003). Knowledge and attitudes about cancer pain management: a comparison of oncology and nononcology nurses. Oncol Nurs Forum, 30, 849-55.

Sterman E, Gauker S, Krieger J (2003). Continuing education: a comprehensive approach to improving cancer pain management and patient satisfaction. Oncol Nurs Forum, 30, 857-64.

Tufekci FG, Ozlu ZK, Arslan s, Gumus K. (2013).Knowledge and attitudes regarding pain management of oncology and non-oncology nurses in Turkey. GARJNM, 2, 1-8.

Wilson B. (2007). Nurses' knowledge of pain. J of Clin Nurs, 16, 1012-20.

Yaakup H, Eng TC, Shah SA (2014). Does clinical experience help oncology nursing staff to deal with patient pain better than nurses from other displines? Knowledge and attitudes survey amongst nurses in a tertiary care in Malaysia. Asian Pac J Cancer Prev, 15, 4885-91.

Yildirim YK, Cicek F, Uyar M (2008). Knowledge and attitudes of Turkish oncology nurses about cancer pain management. Pain Manag Nurs, 9, 17-25. 